\title{
Assessment of stability during gait in patients with spinal deformity-A preliminary analysis using the dynamic stability margin
}

\author{
Anne-Laure Simon ${ }^{\mathrm{a}, \mathrm{b}}$, Vipul Lugade ${ }^{\mathrm{a}, \mathrm{c}}$, Kathie Bernhardt ${ }^{\mathrm{a}}$, A. Noelle Larson ${ }^{\mathrm{d}}$, Kenton Kaufman ${ }^{\mathrm{a}, *}$ \\ ${ }^{a}$ Motion Analysis Laboratory, Mayo Clinic, 200 First Street SW, Rochester, MN 55905, USA \\ b Biomechanics Laboratory, Ecole Nationale Supérieure des Arts et Métiers-Paris Tech, 151 Bd de l'Hôpital, 75013 Paris, France \\ c Whitaker International Program, Department of Physical Therapy, Chiang Mai University, 110 Intawaroroj Rd, Sripoom, Chiang Mai 50200, Thailand \\ d Pediatric Orthopaedics Department, Mayo Clinic, 200 First Street SW, Rochester, MN 55905, USA
}

Keywords:

Gait stability

Spinal deformity

Dynamic stability margin

Extrapolated center of mass

Base of support

\begin{abstract}
A B S T R A C T
Daily living activities are dynamic, requiring spinal motion through space. Current assessment of spinal deformities is based on static measurements from full-spine standing radiographs. Tools to assess dynamic stability during gait might be useful to enhance the standard evaluation. The aim of this study was to evaluate gait dynamic imbalance in patients with spinal deformity using the dynamic stability margin (DSM). Twelve normal subjects and 17 patients with spinal deformity were prospectively recruited. A kinematic 3D gait analysis was performed for the control group (CG) and the spinal deformity group (SDG). The DSM (distance between the extrapolated center of mass and the base of support) and time-distance parameters were calculated for the right and left side during gait. The relationship between DSM and step length was assessed using three variables: gait stability, symmetry, and consistency. Variables' accuracy was validated by a discriminant analysis. Patients with spinal deformity exhibited gait instability according to the DSM $(0.25 \mathrm{~m}$ versus $0.31 \mathrm{~m})$ with decreased velocity ( $1.1 \mathrm{~m} \mathrm{~s}^{-1}$ versus $\left.1.3 \mathrm{~m} \mathrm{~s}^{-1}\right)$ and decreased step length $(0.32 \mathrm{~m}$ versus $0.38 \mathrm{~m})$. According to the discriminant analysis, gait stability was the more accurate variable (area under the curve AUC $=0.98$ ) followed by gait symmetry and consistency. However, gait consistency showed $100 \%$ of specificity, sensitivity, and accuracy of precision. The DSM showed that patients with spinal malalignment exhibit decreased gait stability, symmetry, and consistency besides gait time-distance parameter changes. Additional work is required to determine how to apply the DSM for preoperative and postoperative spinal deformity management.
\end{abstract}

\section{Introduction}

From degenerative scoliosis and kyphosis secondary to osteoporosis in the elderly to spondylolisthesis and scoliosis in young and active individuals, spinal deformities are common and require significant societal resources for treatment. The usual gold standard when quantifying alignment in patients with spinal deformities is based on two-dimensional full-length standing radiographic measurements. The key parameters are numerous and include both spinal and pelvic reference points in the coronal and sagittal planes [1]. However, radiographs do not assess the consequences of such deformities on dynamic balance during gait. Tools to assess the role of dynamic motion and stability are needed to guide clinical treatment. Dynamics in spinal deformities have been reported using data from conventional gait analysis. Patients with scoliosis exhibit modifications of time-distance parameters by reduced velocity and step length as well as asymmetrical ground reaction forces, but no particular modifications of trunk range of motion [2-5]. Dynamic assessment has been recently reported for elderly adults with kyphosis secondary to osteoporosis [6,7]. They showed increased medio-lateral sway and decreased antero-posterior movement of the center of mass (CoM) during gait which is correlated with fall risk [8]. De Groot et al. recently showed similar results according to trunk acceleration smoothness, which was increased in the antero-posterior direction and decreased in the medio-lateral direction [6]. Dynamic balance during gait has not been reported in Scheuermann's disease nor in dysplastic or degenerative spondylolisthesis.

Dynamic stability during gait depends on control of the CoM position and velocity, and on base of support (BoS) displacement combined with proper foot placement [8-10]. On the basis of an inverted pendulum model, Hof et al. suggested a complementary measure for dynamic stability during gait: the extrapolated center of mass $(\mathrm{xCoM})[8,9]$. Using the $\mathrm{xCoM}$, they defined a dynamic stability margin (DSM) to quantify gait dynamic balance. The DSM represents the shortest distance from the $\mathrm{xCoM}$ to the $\mathrm{BoS}$ at all times during the 


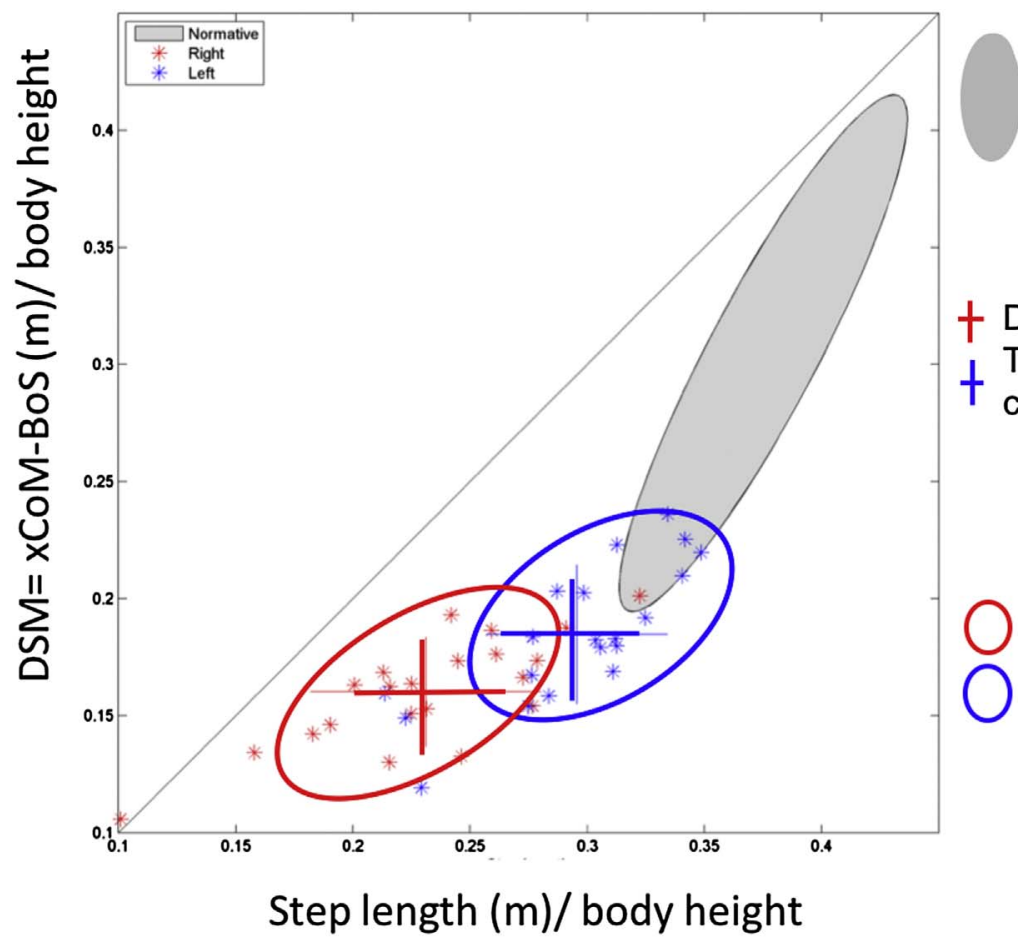

Normative area

If a cross (mean value) is outside this area, the subject is unstable. The grey area define the area of gait stability

\section{Crosses \\ DSM and step length mean values \\ The distance between the blue and red crosses define the symmetry of gait}

\section{Asterisks scatter circle Data at each heel strike collected during the trials The dispersion of the asterisks define the consistency of gait}

Fig. 1. DSM- step length relationship for a 59-year old female with a proximal junctional kyphosis secondary to a T2-S1 fusion for degenerative lumbar scoliosis.

gait cycle. An xCoM located within the BoS during gait indicates gait dynamic stability [10]. Gait instability is therefore defined by an xCoM located outside the BoS.

The aim of this preliminary study was to use the DSM to evaluate the dynamic balance of patients with sagittal and/or coronal spinal deformity. The hypothesis was that patients with spinal deformity would demonstrate reduced gait dynamic stability as shown by the DSM, with greater asymmetry, as evidenced by DSM and step length mean variation between sides, and inconsistency, as evidenced by greater step variability, in their gait when compared to healthy young adults.

\section{Materials and methods}

\subsection{Patient recruitment and selection}

To assess the consequences of spinal deformities on gait dynamic stability, patients undergoing primary or revision spinal surgery were prospectively enrolled between 2011 and 2014 and represent the spinal deformity group (SDG). Patient data were obtained prior to surgery and postoperative evaluation was not available. Inclusion criteria were ambulatory patients with spinal deformity in either the coronal or sagittal plane (scoliosis, kyphoscoliosis, lytic spondylolisthesis, and postoperative flatback). Twelve healthy young adults without spinal deformity constituted the control group (CG). Exclusion criteria for both groups, that might bias the consequences on gait of spinal deformity, included 1) any neurological disease, 2) abnormal gait due to lower limb pathology or injury, and 3) inability to cooperate with gait study. All data were collected after the subject signed an informed consent approved by the Institutional Review Board.

\subsection{Radiographic parameters}

Full-spine bi-planar standing radiographs were performed for the SDG patients. Measurements were performed using Surgimap Spine 2.0 (Nemaris Inc., New York, NY, USA). Sagittal plane measures included pelvic incidence, L1-S1 lordosis, T1-T12 kyphosis, and the sagittal T1 spino-pelvic inclination (T1-SPI). T1-SPI corresponds to the angle between the center of the first thoracic vertebra to the middle of the bicoxo-femoral axis and the vertical reference line [11]. Frontal plane measures the C7-plumbline, which is the angle between the center of the seventh cervical vertebra to the center of the first sacral plate and the vertical reference line [12].

\subsection{Dynamic measures}

Subjects were instructed to walk barefoot at a self-selected comfortable speed along an 8-m walkway. The three-dimensional (3-D) motion of 43 markers placed over bony landmarks was tracked using a tencamera motion capture system operating at $120 \mathrm{~Hz}$ (Motion Analysis Inc, Santa Rosa, CA, USA). Marker-data were low-pass filtered with a fourth order Butterworth filter at a cut-off frequency of $8 \mathrm{~Hz}$. The step length, BoS, and $\mathrm{xCoM}$ were computed from the position of the reflective markers. Calculation of the $\mathrm{xCoM}$ required an estimation of the position and the velocity of the whole-body CoM. A 13-segment rigid body model was used to calculate the weighted-sum of the wholebody CoM (Matlab 8.1 R2013a, Mathworks, Natick, MA, USA) [13]. The boundaries of the BoS were defined using four markers placed on each foot [10]. Clinical measurements used for the DSM calculation were: patient height, trochanteric height, foot length, and width. In accordance with observations that the maximum DSM occurs immediately prior to heel strike, the DSM was calculated from two complete and consecutive heel strikes, separately on the left and right side during three consistent trials [14]. The 3-D coordinates marker data were input into Visual 3D (C-Motion Inc., Rockville, MD, USA) to calculate joint kinematics.

\subsection{Data analysis}

The relationship between the DSM and step length was evaluated (Fig. 1). Both values were normalized to body height. The grey elliptical area represents normative values, which contains all the values collected from the CG.

Three dependent variables were identified for each patient based on the relationship between the DSM and step length: stability, symmetry, and consistency. Patients were classified as positive (stable, symmetric, 
or consistent) or negative (unstable, asymmetric, or not consistent). Stability was defined by the DSM and step length mean values: a subject was classified as unstable if one or both sides mean values were outside the normative area. The distance between left and right side mean DSMstep length coordinates defined symmetry. These mean values are represented by the crosses on Fig. 1. Consistency was defined by the dispersion (asterisks spread out) of the data (DSM and step length) collected during the trials. Each asterisk corresponds to a single value at heel strike.

Five independent variables were calculated in order to assess the reliability of the dependent variables: coordinates distance, median DSM, median step length, standard deviation of DSM, and standard deviation of step length. The coordinates distance was utilized to represent symmetry since a patient with symmetric gait does not have much variation in the DSM and step length values between trials. It was calculated using the Cartesian distance between right and left side mean values coordinates. The median DSM and median step length were calculated using the collected data for each subject in order to avoid any outlier's effect and defined gait stability. The standard deviation of the DSM and the step length were also calculated to represent consistency.

\subsection{Statistical analysis}

All statistical analyses were performed using the JMP 10 software package (SAS Institute Inc., Cary, NC, USA). Numeric data were expressed as mean \pm standard error of the mean (SEM) and confidence intervals (CI95\%). A Shapiro-Wilk test was performed to assess data distribution. Group comparisons were performed with a two-tailed Student $t$-test for the data with normal distribution. A 2-sample Wilcoxon test was performed for the non-parametric data. A p-value of less than 0.05 was considered statistically significant.

Group comparisons for the three dependent variables were performed with a Pearson's Chi-squared test. A stepwise quadratic discriminant analysis with ROC curves was used to validate the use of the graphic method. A model was constructed based on 12 SDG randomly selected patients and the 12 CG subjects. Based on this model, the area under the curve (AUC) was calculated given the ROC curves for each dependent variable. The AUC indicated the probability that a dependent variable misclassified the subject. The closer the AUC was to 1 , the more reliable the dependent variable. Specificity, sensitivity, accuracy, and precision of the model were calculated for the remaining five patients with spinal deformity.

\section{Results}

\subsection{Subject's characteristics}

Seventeen patients with spinal deformities (SDG) were recruited for this preliminary report and had various diagnoses. A common inclusion parameter for spinal deformity was not possible, since the number of subjects for each group was too small. Six had lytic spondylolisthesis (grade III in 3 cases and grade IV in 3 cases). In one case, spondylolisthesis was associated with a mild lumbar scoliosis. Four subjects had scoliosis: degenerative (2), lumbar scoliosis following a thoracic fusion for thoracic adolescent idiopathic scoliosis (1), and congenital lumbar scoliosis secondary to congenital spondylolysis with a S1 agenesis (1). Four subjects had a kyphotic deformity: post-traumatic lumbar kyphosis (1), Scheuermann's disease (1), and junctional kyphosis secondary to spinal fusion (2). One patient had degenerative kypho-scoliosis. Two patients had a flatback syndrome secondary to spinal fusion. For eight patients, primary surgery was performed elsewhere and had come to the institution for revision surgery.

Patients' demographics did not differ between groups except for height (Table 1). Since gait variables for the dynamic stability were normalized to body height, the height difference did not interfere with
Table 1

Subjects demographics. Data are mean \pm SEM and CI 95\%.

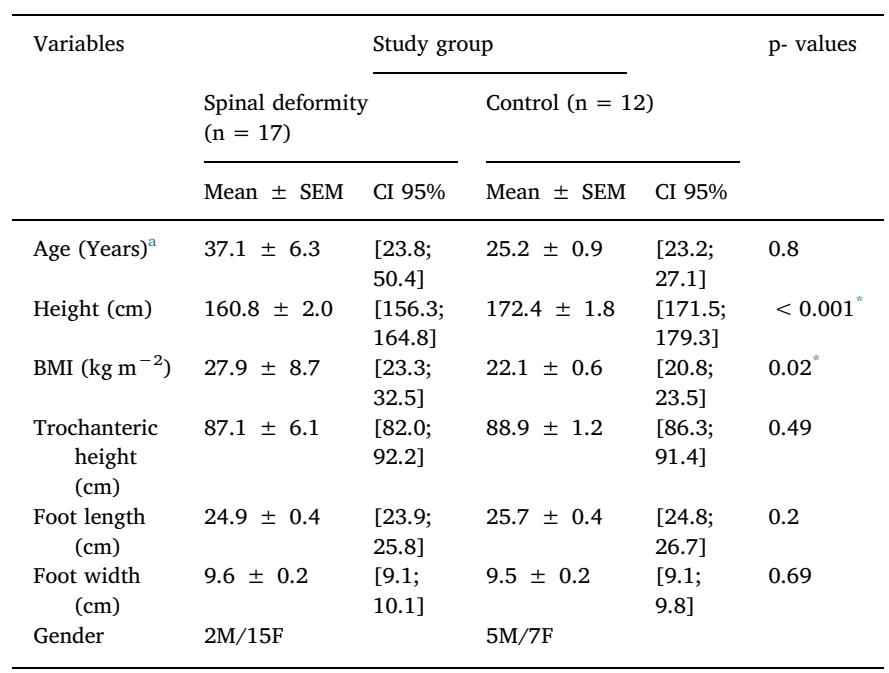

* Significant at $\mathrm{p}<0.05$.

a Non parametric distribution of the data according to Shapiro-Wilk test.

data comparisons.

\subsection{Radiographic parameters}

Patients of the SDG had increased pelvic incidence $\left(64.1^{\circ} \pm 34.5^{\circ}\right)$ and pelvic tilt $\left(27.4^{\circ} \pm 3.3^{\circ}\right)$ compared to previously published reports of normal individuals $[15,16]$ (Fig. 2). Global parameters showed an anterior T1-SPI $\left(3.6^{\circ} \pm 0.9^{\circ}\right)$ (Fig. 2). Mean lordosis and kyphosis were within normal values respectively at $46.5^{\circ} \pm 38.7^{\circ}$ and $40.1^{\circ} \pm 36.4^{\circ}$. Mean C7-plumbine was within normal values $\left(2.4^{\circ} \pm 0.4^{\circ}\right)$.

\subsection{Dynamic data}

Gait characteristics differed significantly between the groups (Table 2). Subjects with spinal deformity had a reduced walking velocity $(\mathrm{p}=0.017): 1.1 \pm 0.1$; CI $95 \%$ [0.9;1.3] vs. $1.3 \pm 0.04$; CI95\% $[1.2 ; 1.4]$ for the SDG and the CG respectively. According to the coordinates distance, they also showed decreased symmetry. Step length was shorter and DSM smaller in the SDG. However, step length was positively influenced by walking velocity. Fourteen patients were unstable according to the values located outside of the normative area. The remaining 3 patients were stable: degenerative kyphoscoliosis (1), thoracic kyphosis secondary to Scheuermann's disease (1), and grade IV lytic spondylolisthesis (1).

\subsection{Stability, symmetry, and consistency}

According to independent variables, patients with spinal deformity were significantly unstable compared to the CG ( $<<0.05)$. SDG patients were also significantly less consistent and less symmetric.

According to dependent variables, the most predictable parameter was gait stability with the highest AUC rate (Table 3). Model sensitivity was low (0.5) except for gait consistency. The model showed $100 \%$ specificity and precision for gait stability and consistency. Perfect accuracy was found for gait consistency.

\section{Discussion}

Daily living activities are dynamic, requiring motion through space. One of the primary goals of spinal deformity surgery is to restore spinal alignment. However, current assessment of spinal balance is only based on static measurements from full-spine standing radiographs. 


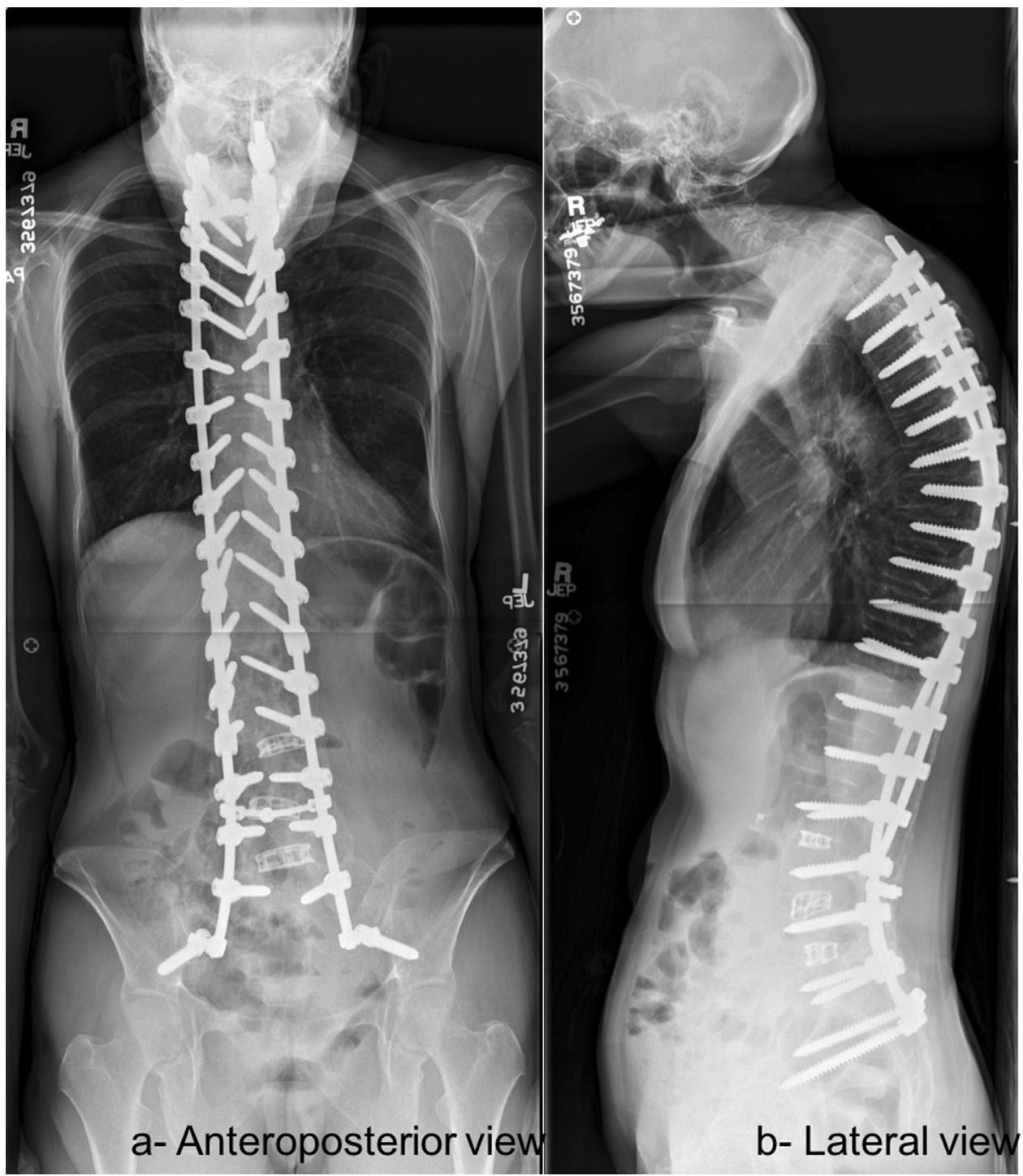

Fig. 2. Bi-planar radiographs from the 59-year old female with proximal junctional kyphosis.

Compared to literature values for asymptomatic subjects, radiographic measurements showed that the subjects of this study exhibited spinal misalignment $[12,15,16]$. Tools to assess spine dynamic motion and stability during gait may be useful to complement the standard radiographic evaluation. Previously, several measurements have been used to quantify gait dynamic stability [17-19]. However, none of these measurements have been specifically applied to patients with spinal deformity. The analysis could be interesting for the assessment of gait development in younger patients affected by spinal deformity and serve as a surgical planning tool to predict the necessary amount of surgical deformity correction [20-22]. Furthermore, this analysis could be particularly useful in the elderly with degenerative spinal disease who are at increased risk of falls. Two studies have considered fall risk in the elderly with sagittal spinal deformities [6,7]. Although the fall

Table 2

Gait variables including results from the 5 independent variables. Data are mean \pm SEM and CI 95\%.

\begin{tabular}{|c|c|c|c|c|c|c|}
\hline \multirow[t]{3}{*}{ Variables } & & & \multicolumn{2}{|l|}{ Study group } & & \multirow[t]{3}{*}{ p- values } \\
\hline & & \multicolumn{2}{|c|}{ Spinal deformity $(\mathrm{n}=17)$} & \multicolumn{2}{|l|}{ Control $(\mathrm{n}=12)$} & \\
\hline & & mean \pm SEM & CI 95\% & mean \pm SEM & CI $95 \%$ & \\
\hline Velocity $\left(\mathrm{m} \mathrm{s}^{-1}\right)^{\mathrm{a}}$ & & $1.1 \pm 0.1$ & {$[0.9 ; 1.3]$} & $1.3 \pm 0.04$ & {$[1.2 ; 1.4]$} & $0.017^{*}$ \\
\hline Symmetry & Right-to-left foot distance (m) ${ }^{a}$ & $0.05 \pm 0.01$ & {$[0.03 ; 0.07]$} & $0.02 \pm 0.002$ & {$[0.02 ; 0.03]$} & $0.008^{k}$ \\
\hline \multirow[t]{2}{*}{ Stability } & Median step length (m) & $0.32 \pm 0.02$ & {$[0.28 ; 0.35]$} & $0.38 \pm 0.007$ & {$[0.36 ; 0.39]$} & $0.002^{*}$ \\
\hline & Median DSM (m) & $0.25 \pm 0.02$ & {$[0.19 ; 0.29]$} & $0.31 \pm 0.01$ & {$[0.28 ; 0.33]$} & $0.04^{*}$ \\
\hline \multirow[t]{2}{*}{ Consistency } & Step length $\mathrm{SD}^{\mathrm{a}}$ & $0.02 \pm 0.004$ & {$[0.01 ; 0.03]$} & $0.01 \pm 0.001$ & {$[0.008 ; 0.012]$} & $0.01^{*}$ \\
\hline & DSM SD & $0.022 \pm 0.003$ & {$[0.02 ; 0.03]$} & $0.016 \pm 0.002$ & {$[0.01 ; 0.02]$} & $0.04^{*}$ \\
\hline
\end{tabular}

SD: Standard deviation.

* Significant at $\mathrm{p}<0.05$.

${ }^{a}$ Non-parametric data using 2-samples Wilcoxon test. 
Table 3

Receiver Operating Characteristic (ROC) Analysis of Dependent Variables.

\begin{tabular}{llll}
\hline Variables & Stability & Symmetry & Consistency \\
\hline Area under the curve & 0.98 & 0.82 & 0.69 \\
Specificity & 0.50 & 0.50 & 1.00 \\
Sensitivity & 0.86 & 0.71 & 0.75 \\
Accuracy & 0.78 & 0.67 & 0.78 \\
\hline
\end{tabular}

Percentages values expressed in decimal form.

risk scoring system was different, both studies showed that trunk acceleration was more variable compared to normal subjects. Gait pattern was related to difficulties in counterbalancing the forces in order to realign their CoM during gait. Therefore, spinal deformity patients were found to have increased propensity to fall, which is concordant to the results of this study.

The DSM used in this current study was a function of the CoM location, CoM velocity, and of the BoS. Therefore, this tool was of particular interest because it was a direct measure of gait stability. The maximum Lyapunov exponent and tri-axial accelerometry had also been used to quantify gait dynamic balance but only based on interindividual variability of gait patterns $[17,21]$.

\subsection{Dynamic data}

Several authors reported changes occurring during gait in patients with scoliosis or low back pain [23-26]. Time-distance parameters, kinematic ranges of motion, ground reaction forces, and dynamic electromyography data are modified in both pathologies. A recent study by Paul et al. used analysis of the CoM and center of pressure (CoP) displacements in patients with adolescent idiopathic scoliosis [24]. The aim was to report postoperative improvement of stability during gait. They showed coronal plane reduced CoM sway. However, their study did not illustrate if conservative strategies for better stability were adopted, since foot position and BoS were not examined.

Accordingly, compared to normal controls, this study showed that patients with spinal deformity had a significantly lower DSM. Patients also had shorter step lengths and slower velocity. Other authors have attempted to correlate velocity and DSM $[27,28]$. Hak et al. reported a positive association between the DSM and walking speed for normal subjects, such that an increased walking speed was associated with an increased DSM value in the antero-posterior direction [28]. Their study was conducted on a treadmill, which might have influenced the results [27]. Furthermore, determination of the whole-body CoM was based on a single sacral marker. Gard et al. demonstrated that with faster velocity, the sacral marker method produced greater excursion than with segmental analysis, which was used in this study [29]. Thus, further work needs to be done to evaluate the effect of walking velocity on the DSM.

\subsection{Stability, symmetry, and consistency}

Currently, assessment of gait stability by our institution is based on gait visual inspection and on the DSM to step length relationship graphic (Fig. 1). There is no range of normative values for this relationship. Therefore, a validation of the graphic method was necessary. This was provided by the discriminant analysis, which used quantitative variables calculated from the gait analysis data. The graph contained three dependent key parameters: stability, symmetry, and consistency. According to the ROC curves (Table 3), stability was the most accurate variable followed then by symmetry and consistency. However, $100 \%$ of specificity was identified for gait consistency. Previous reports have evaluated sagittal and frontal plane DSM excursion $[7,28]$. However, in the present study, various spinal deformities affecting sagittal and frontal radiological parameters were analyzed.
Therefore, bi-planar assessment of the DSM was not considered as relevant.

\section{Limitations}

This study aimed to report preliminary results and therefore had several limitations. First, the main limitation was the wide variety of diagnoses for the study cohort. Readers must take into account that these are preliminary results. The authors aimed to introduce the DSM. Even tested on a limited and heterogeneous population of patients, results showed significant dynamic differences between cases and controls. Subgroup analyses were attempted according to the different spinal pathologies, but results were not relevant due to the small sample size. Establishing a specific pattern of gait dynamic stability for each type of spinal deformity and their relationship to radiographic measurements requires further investigation with a larger and more homogeneous sample. Second, there were a limited number of subjects for the SDG without matching controls. There was no significant difference for the demographics between groups except for the height, which was a normalized variable for the DSM calculation (Table 1). Further investigation with a larger group and matched controls would provide more precise sensitivity, specificity, accuracy, and precision. Third, an assessment of the inter-session DSM repeatability in the SDG was not performed. However, the DSM was based on kinematic measurements, upon which repeatability was regularly performed at the laboratory. Finally, the walking speed was not controlled in this study. All subjects walked at their self-selected walking velocity which reflects how they would ambulate in the free-living environment. There was a difference in the walking speed between the two groups. A sensitivity analysis demonstrated that this difference in walking speed did not affect the conclusions reached regarding the DSM. This is due to the fact that the $\mathrm{xCoM}$ includes the subject's walking velocity as a component of the calculation for the DSM.

\section{Conclusions}

In summary, gait dynamic stability was altered in patients with spinal deformity compared to controls. In this study, the relationship between the DSM and step length permitted the validation of a model using normal and spinal deformity subjects. Patients with spinal deformity had decreased gait stability, symmetry, and consistency. Additional work is required to determine how to leverage these findings to perform effective preoperative planning for spinal deformity correction. This is a promising area of work to analyze complementary measures (static and dynamic) in spinal deformity. This understanding could help surgeons to better plan surgery and deformity correction in order to optimize patient outcomes and balance.

\section{Conflicts of interest}

There is no financial or personal relationship to disclose, nor any other conflicts of interest, that may bias or influence this study.

\section{References}

[1] G. Duval-Beaupere, C. Schmidt, P. Cosson, A barycentremetric study of the sagittal shape of spine and pelvis: the conditions required for an economic standing position, Ann. Biomed. Eng. 20 (1992) 451-462.

[2] N. Chockalingam, P.H. Dangerfield, A. Rahmatalla, N. Ahmed el, T. Cochrane, Assessment of ground reaction force during scoliotic gait, Eur. Spine J. 13 (2004) $750-754$.

[3] G. Giakas, V. Baltzopoulos, P.H. Dangerfield, J.C. Dorgan, S. Dalmira, Comparison of gait patterns between healthy and scoliotic patients using time and frequency domain analysis of ground reaction forces, Spine 21 (1996) 2235-2242.

[4] I.A. Kramers-de Quervain, R. Muller, A. Stacoff, D. Grob, E. Stussi, Gait analysis in patients with idiopathic scoliosis, Eur. Spine J. 13 (2004) 449-456.

[5] P. Mahaudens, X. Banse, M. Mousny, C. Detrembleur, Gait in adolescent idiopathic scoliosis: kinematics and electromyographic analysis, Eur. Spine J. 18 (2009) $512-521$. 
[6] M.H. de Groot, H.C. van der Jagt-Willems, J.P. van Campen, W.F. Lems, J.H. Beijnen, C.J. Lamoth, A flexed posture in elderly patients is associated with impairments in postural control during walking, Gait Posture 39 (2014) 767-772.

[7] M. Sinaki, R.H. Brey, C.A. Hughes, D.R. Larson, K.R. Kaufman, Balance disorder and increased risk of falls in osteoporosis and kyphosis: significance of kyphotic posture and muscle strength, Osteoporos. Int. 16 (2005) 1004-1010.

[8] A.L. Hof, M.G. Gazendam, W.E. Sinke, The condition for dynamic stability, J. Biomech. 38 (2005) 1-8.

[9] A.L. Hof, The 'extrapolated center of mass' concept suggests a simple control of balance in walking, Hum. Mov. Sci. 27 (2008) 112-125.

[10] V. Lugade, V. Lin, L.S. Chou, Center of mass and base of support interaction during gait, Gait Posture 33 (2011) 406-411.

[11] V. Lafage, F. Schwab, A. Patel, N. Hawkinson, J.-P. Farcy, Pelvic tilt and truncal inclination: two key radiographic parameters in the setting of adults with spinal deformity, Spine 34 (2009) E599-E606.

[12] J.-M. Mac-Thiong, P. Roussouly, E. Berthonnaud, P. Guigui, Sagittal parameters of global spinal balance: normative values from a prospective cohort of seven hundred nine caucasian asymptomatic adults, Spine 35 (2010) E1193-E1198.

[13] D.A. Winter, Center of Mass of a Multi-segment System. Biomechanics and Motor Control of Human Movement, 4th ed., John Wiley \& Sons, Inc., Hoboken, New Jersey, 2009p. 88

[14] A.L. Hof, The equations of motion for a standing human reveal three mechanisms for balance, J. Biomech. 40 (2007) 451-457.

[15] J.-M. Mac-Thiong, H. Labelle, E. Berthonnaud, R.R. Betz, P. Roussouly, Sagittal spinopelvic balance in normal children and adolescents, Eur. Spine J. 16 (2007) 227-234.

[16] R. Vialle, N. Levassor, L. Rillardon, A. Templier, W. Skalli, P. Guigui, Radiographic analysis of the sagittal alignment and balance of the spine in asymptomatic subjects, J. Bone Joint Surg. Am. 87-A (2005) 260-267.

[17] S.M. Bruijn, O.G. Meijer, P.J. Beek, J.H. van Dieen, Assessing the stability of human locomotion: a review of current measures, J. R. Soc. Interface 10 (2013) 20120999.
[18] Y. Hurmuzlu, C. Basdogan, On the measurement of dynamic stability of human locomotion, J. Biomech. Eng. 116 (1994) 30-36.

[19] R. Moe-Nilssen, A new method for evaluating motor control in gait under real-life environmental conditions. Part 1: the instrument, Clin. Biomech. 13 (1998) 320-327.

[20] F.B. Horak, C.L. Shupert, A. Mirka, Components of postural dyscontrol in the elderly: a review, Neurobiol. Aging 10 (1989) 727-738.

[21] J.J. Kavanagh, H.B. Menz, Accelerometry: a technique for quantifying movement patterns during walking, Gait Posture 28 (2008) 1-15.

[22] P.W. Overstall, A.N. Exton-Smith, F.J. Imms, A.L. Johnson, Falls in the elderly related to postural imbalance, Br. Med. J. 1 (1977) 261-264.

[23] C. Fortin, S. Nadeau, H. Labelle, Inter-trial and test-retest reliability of kinematic and kinetic gait parameters among subjects with adolescent idiopathic scoliosis, Eur. Spine J. 17 (2008) 204-216.

[24] J.C. Paul, A. Patel, K. Bianco, E. Godwin, Q. Naziri, S. Maier, et al., Gait stability improvement after fusion surgery for adolescent idiopathic scoliosis is influenced by corrective measures in coronal and sagittal planes, Gait Posture 40 (2014) 510-515.

[25] M.J. Simmonds, C.E. Lee, B.R. Etnyre, G.S. Morris, The influence of pain distribution on walking velocity and horizontal ground reaction forces in patients with low back pain, Pain Res. Treat. 2012 (2012) 214980.

[26] L. Vogt, K. Pfeifer, M. Portscher, W. Banzer, Influences of nonspecific low back pain on three-dimensional lumbar spine kinematics in locomotion, Spine 26 (2001) 1910-1919.

[27] J.B. Dingwell, L.C. Marin, Kinematic variability and local dynamic stability of upper body motions when walking at different speeds, J. Biomech. 39 (2006) 444-452.

[28] L. Hak, H. Houdijk, P.J. Beek, J.H. van Dieen, Steps to take to enhance gait stability: the effect of stride frequency, stride length, and walking speed on local dynamic stability and margins of stability, PLoS One 8 (2013) e82842.

[29] S.A. Gard, S.C. Miff, A.D. Kuo, Comparison of kinematic and kinetic methods for computing the vertical motion of the body center of mass during walking, Hum. Mov. Sci. 22 (2004) 597-610. 